\title{
BMJ Open PACMAN trial protocol, Perioperative Administration of Corticotherapy on Morbidity and mortality After Non- cardiac major surgery: a randomised, multicentre, double-blind, superiority study
}

Karim Asehnoune, ${ }^{1}$ Emmanuel Futier, ${ }^{2}$ Fanny Feuillet, ${ }^{3,4}$ Antoine Roquilly, ${ }^{5}$ For the PACMAN group

To cite: Asehnoune K, Futier E, Feuillet $\mathrm{F}$, et al. PACMAN trial protocol, Perioperative Administration of Corticotherapy on Morbidity and mortality After Non-cardiac major surgery: a randomised, multicentre, double-blind, superiority study. BMJ Open 2019;9:e021262. doi:10.1136/ bmjopen-2017-021262

- Prepublication history and additional material for this paper are available online. To view these files, please visit the journal online (http://dx.doi. org/10.1136/bmjopen-2017021262).

Received 22 December 2017 Revised 4 January 2019 Accepted 8 January 2019
D) Check for updates

(c) Author(s) (or their employer(s)) 2019. Re-use permitted under CC BY-NC. No commercial re-use. See rights and permissions. Published by BMJ.

For numbered affiliations see end of article.

\section{Correspondence to}

Professor Karim Asehnoune; karim.asehnoune@chu-nantes. fr

\section{ABSTRACT}

Introduction Postoperative complications are major healthcare problems and are associated with a reduced short-term and long-term survival after surgery. An excessive postoperative inflammatory response participates to the development of postoperative infection and mortality. The aim of the Perioperative Administration of Corticotherapy on Morbidity and mortality After Non-cardiac surgery (PACMAN) study is to assess the effectiveness of perioperative administration of corticosteroid to reduce postoperative morbidity and mortality in patients undergoing major noncardiac surgery.

Methods and analysis The PACMAN is a multicentre, randomised, controlled, double-blind, superiority, twoarm trial of 1222 high-risk patients aged 50 years or older undergoing major non-cardiac surgery at 32 acute care hospital in France. Patients are randomly assigned to dexamethasone $(0.2 \mathrm{mg} / \mathrm{kg}$ at the end of the surgical procedure and at day $+1, n=611)$ or to placebo $(n=611)$. The primary outcome is a composite of predefined 14-day major pulmonary complications and mortality. Secondary outcomes are surgical complications, infections, organ failures, critical care-free days, length of hospital stay and all-cause mortality at 28 days.

Ethics and dissemination The PACMAN trial protocol has been approved by the ethics committee of Sud Mediterranée $\mathrm{V}$, and will be carried out according to the Good Clinical Practice guidelines and the principles of the Declaration of Helsinki. The PACMAN trial is a randomised controlled trial powered to investigate whether perioperative administration of corticosteroids in patients undergoing non-cardiac major surgery reduces postoperative complications. The results of this study will be disseminated through presentation at scientific conferences and publication in peer-reviewed journals.

Trial registration number NCT03218553; Pre-results.

\section{INTRODUCTION}

More than 300 million major surgical procedures are undertaken worldwide each year. ${ }^{1}$
Strengths and limitations of this study

- This study is a multicentre, randomised, controlled and double-blind trial adequately powered to determine whether corticosteroid reduces postoperative complications in high-risk patients undergoing major non-cardiac surgery.

- Potential treatment's benefits include reduced risk of postoperative infection, development of organ failure and reduced risk of mortality.

- Limitations due to the difficulty of sepsis diagnosis after major surgery are limited by the use of a placebo ensuring a double-blind evaluation of the primary outcome.

- This large study has the potential of changing international recommendations on the management of high-risk patients undergoing major non-cardiac surgery.

For most patients, risks of surgery are low. However, in an European international cohort, the mortality rate for patients undergoing non-cardiac surgery was higher than excepted (4\% of patients died before hospital discharge). ${ }^{2}$ It is interesting to consider that $10 \%$ of patients at risk of postoperative complications represent $80 \%$ of postoperative deaths. ${ }^{3}$ It is also important to consider that patients who develop complications but survive until hospital discharge have usually reduced functional independence and long-term survival. ${ }^{4}$ These data suggest that interventions to prevent complications and mortality should probably be undertaken early. One of the main targets to focus on is probably lung. Indeed, postoperative respiratory complications represent the most common perioperative complication after 
wound infections with an estimated incidence ranging from $2.0 \%$ to $5.6 \%$ for surgical procedures. ${ }^{4}$ Respiratory failure after general anaesthesia and tracheal extubation has been shown to be one of the most meaningful factors associated with poor patient outcomes, leading to longer hospital stay. ${ }^{4}$ Considering, the high volume of surgical procedures undertaken each year, the key message is that decreasing even a low rate of avoidable harm will be associated with a high-cost saving for the society and many preventable deaths and complications for the patients.

Major surgery induces an inflammatory response characterised by activation of platelets, neutrophils, monocytes, macrophages, cascades (coagulation, fibrinolytic and kallikrein). The consequences of tissue surgical injuries are a release of danger-associated molecular patterns which initiate the production of proinflammatory mediators (cytokines, radical oxygen species). ${ }^{5}$ This inflammatory response is useful for tissue healing, but it is thought that an excessive response contributes to postoperative morbidity (such as infections and organ failures) and mortality. Glucocorticoids have thus been proposed to reduce the risk of complications in several medical conditions characterised by systemic inflammatory response.

In patients undergoing major cardiac surgery, corticosteroids were associated with reduction in length of intensive care unit (ICU) stay, ${ }^{6}$ but no difference in 30-day mortality or major morbidity in was found. ${ }^{7}$ In patients with severe trauma, considering the potential immunostimulating effects of 'low-doses' corticosteroids, ${ }^{8}$ we have shown, in two multicentre, randomised, doubleblind placebo-controlled study on intubated patients with trauma, that the use of an intravenous low-dose corticosteroids, compared with placebo, resulted in a decreased risk of hospital-acquired pneumonia. ${ }^{9}{ }^{10}$ Interestingly, apart from higher insulin consumption in patients receiving corticosteroids, no significant harm related to treatment was recorded in both studies. In major non-cardiac surgery, a recent meta-analysis concluded that proofs are lacking to demonstrate clinically important benefits with perioperative administration of glucocorticoids, and that safety has not been sufficiently investigated to rule out any clinically important side effects. ${ }^{11}$ The objective of the Perioperative Administration of Corticotherapy on Morbidity and mortality After Non-cardiac surgery (PACMAN) study is to ascertain whether or not the administration of early corticosteroid with standard care compared with standard care alone prevents respiratory complications and reduces mortality in high-risk patients undergoing major surgery. We are reporting the V.4 of the protocol (2 September 2017). This manuscript has been submitted for publication on the 30 November 2017 , before the inclusion of the first patient in the study.

\section{METHODS AND DESIGN Hypothesis}

High-risk patients treated with short course of corticosteroid have reduced morbidity and mortality rates

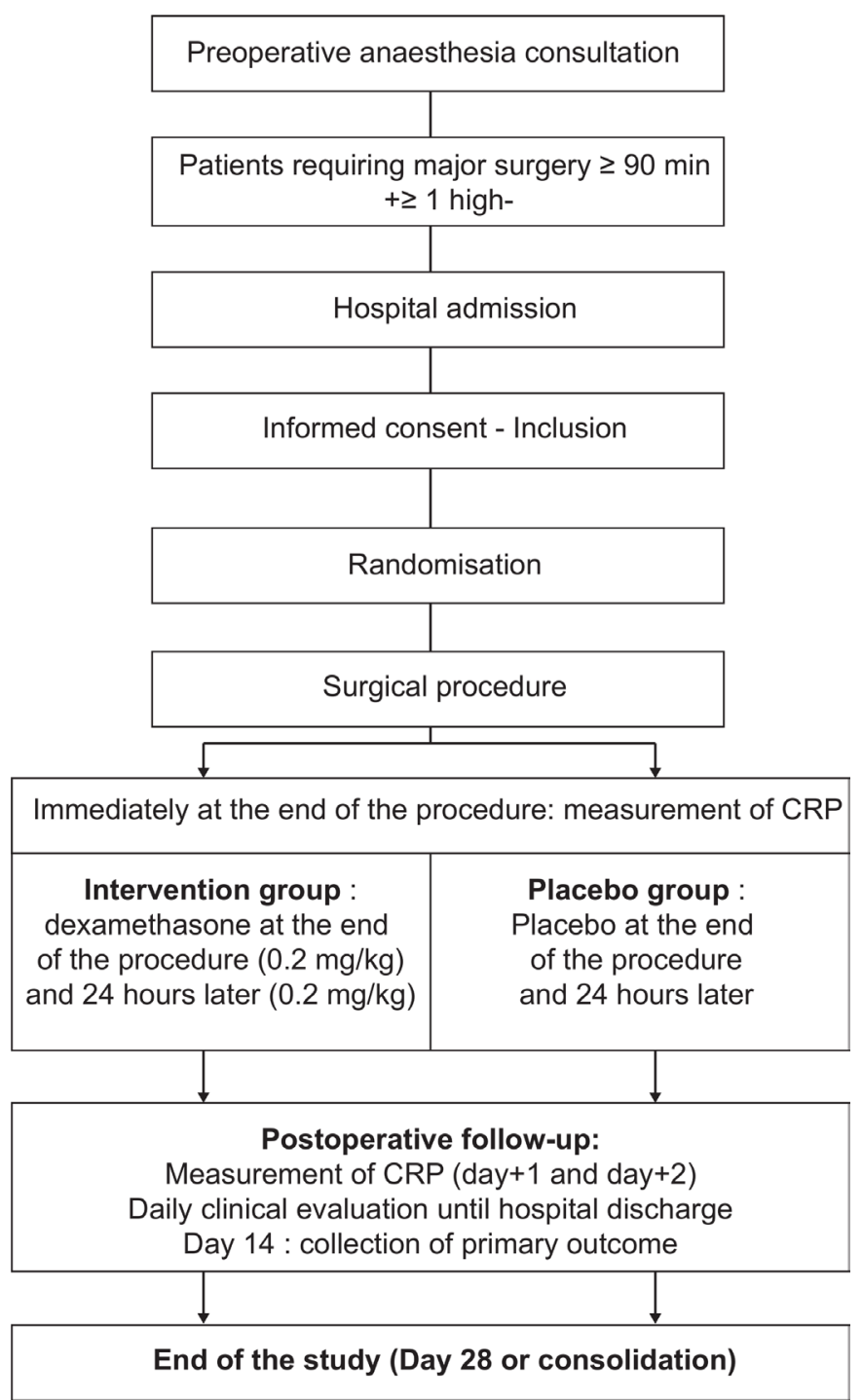

Figure 1 Flow chart. CRP, C reactive protein.

compared with those receiving standard care alone after major non-cardiac surgery.

\section{Research questions}

1. Does short course of moderate doses of dexamethasone prevent death and/or postoperative respiratory complications after major non-cardiac surgery in highrisk patients?

2. Does short course of moderate doses of dexamethasone reduce the duration of hospitalisation after major non-cardiac surgery in high-risk patients?

3. Does short course of moderate doses of dexamethasone prevent delayed skin healing?

\section{Design}

The PACMAN study is a multicentre, randomised, double-blind, parallel-group, superiority, controlled trial (figure 1). KA and AR wrote the first draft of the protocol. FF was responsible for the statistical plan. EF extensively revised the protocol. All the authors approved the final version. 


\section{Ethic approval}

The Institutional Review Board of Sud Mediterranée V (France) approved the study protocol (June 2017, V.4). Patients provided written consent for participation (see online supplementary file). The PACMAN trial is conducted in accordance with the Declaration of Helsinki.

\section{Setting}

The study involved 33 French hospitals, each centre caring for more than 200 patients undergoing major surgery each year.

\section{Study population}

Investigators screen consecutive high-risk patients undergoing major non-cardiac surgery. Patients older than 50 years and scheduled for a major surgery $(>90 \mathrm{~min}$ and performed under general anaesthesia) of the abdomen, pelvis, thorax, face/neck, vascular surgery are eligible provided that they have one or more of the following risk factors: age $>65$ years, presence of a defined risk factor for cardiac or respiratory disease (exercise tolerance equivalent to 6 metabolic equivalents or less), medical history of stroke, moderate to severe renal impairment (clearance of creatinine $\leq 30 \mathrm{~mL} / \mathrm{L}$ ), active smoking, averaged observed blood losses over $500 \mathrm{~mL}$ or emergency surgery. These risk factors have been adapted from the systematic review for the preoperative pulmonary risk stratification for non-cardiothoracic surgery published by the American College of Physicians ${ }^{12}$ and from the guidelines for the management of severe perioperative bleeding of the European Society of Anaesthesiology. ${ }^{13}$ Exclusions criteria are: allergy to the intravenous formulation of dexamethasone, treatment with systemic corticosteroids at a dose $>5 \mathrm{mg}$ /day of equivalent prednisolone in the previous 3 months, uncontrolled psychotic disorder (acute or chronical) chronic renal failure (clearance of creatinine $<10 \mathrm{~mL} / \mathrm{min}$ ), life expectancy of less than 1 month, preoperative shock (defined by the need for continuous infusion of vasoactive drugs (norepinephrine, epinephrine or dobutamine), acute pulmonary oedema in the last 7 days, active bacterial or viral infection, pregnant women, breastfeeding women, minors, adults under guardianship or trusteeship.

\section{Identification and information of patients}

All consecutive adult patients requiring surgery with an expected duration $\geq 90 \mathrm{~min}$ will be assessed for eligibility. During the anaesthesia consultation or in the operating room in case of emergency surgery, local investigators (anaesthesiologists and/or surgeons) will verify inclusion and exclusion criteria. Investigators will invite the patients to participate in the study. Patients will be informed in complete and faithful terms and in understandable language of the objectives and constraints of the study, the potential risks, the required observation and safety measures, and their right to refuse to participate in the study or to revoke their consent at any time. All information appears in an information notice and consent form given to the patient. Written informed consent will be obtained by the investigator. All these documents are approved by the competent ethics committee. Two original copies will be cosigned by both the investigator and the patient and the parents. The second copy is to be kept in the patient's medical record.

\section{Treatment allocation}

Patients are randomised in a 1:1 ratio and stratified according to cancer (yes/no) and according to the type of surgical procedure (thoracic surgery or not). Randomisation is made by a computerised number generator list provided by a statistician not involved in the determination of eligibility or in the assessment of outcomes. All assignments are made through a dedicated, password-protected, SSL-encrypted website. Patients are randomised in the first 24 prior to surgery to dexamethasone (intervention group) or to placebo (control group).

\section{Masking protocol}

Randomised patients are given a number corresponding to a «PACMAN treatment pack» that contains: $4 \times 10 \mathrm{mg}$ vial of dexamethasone or and a sheet for schedule administration.

\section{Procedures}

At the end of the surgery ( $<2$ hours after skin closure), and before study drug administration, the blood level of $\mathrm{C}$ reactive protein (CRP) is measured (figure 1). Then, patients randomised to either slow intravenous infusion of dexamethasone $(0.2 \mathrm{mg} / \mathrm{kg}$ of real body weight within the 2 hours after the end of the surgery and at day +1 ) or injection of placebo receive treatment (figure 1). The blood level of CRP is measured at day +1 and day +2 after surgery. The treatment is administrated slowly (over 15-30 min) independently of the CRP levels.

\section{Standard of care}

According to recent recommendations and publications, clinicians are prompted to realise adequate timing of antimicrobial prophylaxis, ${ }^{14}$ to apply a protective ventilation strategy (low tidal volume and positive-end expiratory pressure) during surgery, ${ }^{15}$ to closely monitor and treat perioperative hypovolaemia and hypotension ${ }^{16} 17$ and to early stop sedation at the end of the surgery. ${ }^{18}$ Decisions of postoperative admission to ICU and of prophylactic application of non-invasive ventilation will follow local standard of cares. Prophylactic administration of glucocorticoid for postoperative nausea and vomiting or postoperative oedema is not permitted. Clinicians can use glucocorticoid during the first 7 days of the study only in case of formal indication of steroid such as stridor (rescue therapy).

\section{Protocol drop-out}

For patients developing an allergic reaction to the study treatment, the second injection will not be performed and the appropriate treatment of the allergic reaction will 
be provided. Patients with allergic reaction to dexamethasone will be kept in analysis and remain analysis with the intervention group. Patients treated with out-of-protocol glucocorticoid will be kept in analysis and remain analysis with their attributed group. No procedure for revealing a participant's allocated intervention during the trial is planned.

\section{Study endpoints}

The primary outcome is a composite outcome (all-cause mortality and major postoperative complications) within 14 days after surgery, at least one item among the following: postoperative sepsis, postoperative pulmonary complication (postoperative pneumonia, need for invasive ventilation and/or non-invasive ventilation for respiratory failure) and all-cause mortality.

The diagnosis of pneumonia will be retained according to European guidelines, that is, association 48 hours after admission of at least 2 signs (body temperature $>38^{\circ} \mathrm{C}$; leucocytosis $>12$ ' $\mathrm{x} 10^{9} / \mathrm{L}$ or leucopenia $<4$ ' $\mathrm{x} 10^{9} \mathrm{~L}$; purulent pulmonary secretions) with the appearance of a new infiltrate or change in an existing infiltrate on chest X-ray and a bacterial documentation (blood or respiratory fluid analysis). ${ }^{19} 20$

The rates of patients discharged before day 14, and evaluated between day 14 and day 28, will be reported. Secondary outcomes are all-cause mortality at 28 days, duration of invasive mechanical ventilation, duration of non-invasive mechanical ventilation, hospital free-days at 28 days, surgical complications according to the ClavienDindo classification within 28 days, unplanned admission or readmission to ICUs (within 28 days following randomisation), organ failures within 14 days after surgery, Sequential Organ Failure Assessment at day +1 and day +3 , proportion of patients who experienced adverse events, especially hyperglycaemia, healing impairment, anastomotic leakage at day +14 after surgery.

\section{Follow-up data}

The following variables are collected: demographics, American Society of Anesthesiology score, preoperative medical optimisation, per-surgery management (drugs, mechanical ventilation, durations of procedure, fluid infusion, bleeding, postoperative analgesia), infections, organ failures, blood levels of CRP, healing, postoperative complications (including anastomotic leakage), length of ventilator support and ICU hospitalisation and death at day 28 are recorded.

\section{Data collection and checking}

Data will be entered into the electronic web-based (Clinsight) case report form (eCRF) by trial or clinical personnel under the supervision of the study site investigators. From the eCRFs, the trial database will be established.

\section{Study monitoring}

The study will be monitored on behalf of the promotor (Nantes University Hospital). Site staff will be available to facilitate the monitoring visits and ensure that all required documentation is available for review. Study initiation visits are carried out at all sites before recruitment starts at that site. During regular monitoring visits realised throughout the duration of the trial, an independent research assistant will carry out Source Data Verification of trial data, verify informed consent forms and ensure the completeness of the investigator site files.

\section{Study oversight}

Study sponsor is the Nantes University Hospital (5 allée de l'ile Gloriette, 44000 Nantes, drc-nantes@chu-nantes. fr). Experienced research staff will monitor the study for quality, the integrity of data in all the participating centres. Serious adverse events and unexpected related or possibly related serious events are reported blinded to the promotor within respectively 24 hours or 7 days. An independent data and safety monitoring board (DSMB) is appointed by the sponsor. The DSMB is made up of four individuals with no connection to the research, including three clinicians specialising in the management of ICU patients and corticotherapy in ICU, and a methodologist/biostatistician. Before the first inclusion and every 600 inclusions, the DSMB looks over the ethics in accordance with the Declaration of Helsinki, monitors patient safety and reviews safety issues as the study progresses. The DSMB makes recommendations to the sponsor about the continuation, modification or termination of the research. The recommendations that the DSMB can make are:

- To continue the research with no modifications.

- To continue the research with a modification to the protocol and/or to the monitoring of subjects.

- To temporarily halt inclusions.

- To permanently terminate the research in light of serious adverse reactions.

- To recommend increasing the total number of patients to be included to ensure the study power in case of high number of exclusions in the per-protocol analysis.

The DSMB has a consultative role in advising the sponsor on safety issues such as tolerance and reassessment of the benefit:risk ratio during the research. Trial recruitment can be stopped by the promotor on the advice of the DSMB in case of safety concern.

\section{Statistical consideration}

All analyses will be performed with the use of SAS software (V.9.4) before the breaking of the randomisation code, according to International Conference on Harmonisation-Good Clinical Practice guidelines. Analyses will be conducted, first, on data from the modified intention-totreat (mITT) population, second, in the ITT population as well as in the per-protocol population. The criteria for including patients in the mITT and in the per-protocol populations, respectively, are provided below.

Continuous variables will be presented as mean and SDs (as median and quartiles, otherwise) and will be 
compared with the use of the unpaired t-test or the MannWhitney U test when appropriate. The Shapiro-Wilk test will be used to assess normality, and the Fisher-Snedecor test to assess homoscedasticity. Categorical data will be presented as exact numbers and percentages.

\section{Number of patients}

The rate of the primary endpoint in the control group is expected to reach 20\%. ${ }^{15}{ }^{21}$ Assuming a 20\% rate in the control group and $14 \%$ in the dexamethasone group, a total of 1222 patients are needed to detect this difference between the two groups with a $5 \%$ type I error and a power of $80 \%$ in a two-sided test.

\section{Preplanned primary analysis}

For the primary analysis, data will be analysed with the use of logistic regression adjusted for stratification factors (cancer and thoracic procedure).

The effects of the treatment will be investigated in ITT, in mITT and in per-protocol populations. In the ITT analyse, all randomised patients will be kept in analysis. In the mITT analyse, all randomised patients will be kept in analysis except those who would not have been eligible for randomisation according to the inclusion/ non-inclusion criteria or those who have not received any injection of the experimental treatment (dexamethasone or placebo). In the per-protocol analyse, all randomised patients will be kept in analysis except patients having one or more major protocol violations defined as those who would not be eligible for randomisation according to inclusion/non-inclusion criteria; or those who accidentally would have received the wrong intervention (dexamethasone or placebo); or those in whom surgical intervention could not have been done (eg, intra-abdominal extensive cancer); or those who have withdrawn consent; those who would have received out-of-protocol glucocorticoids.

\section{Prespecified exploratory subgroup analyses}

- States of randomisation (cancer yes/no, thoracic procedure yes/no).

- Medical history of corticosteroid in the preceding 3 months (yes/no).

- Emergency versus scheduled surgery.

- According to the level of CRP measured at the end of the surgical procedure immediately before the first injection of the studied treatment $(<50,50-150$ or $>150 \mathrm{mg} / \mathrm{mL}$ ).

- According to the surgery (abdomen, pelvis, thorax, face/neck or vascular surgery).

- In diabetic and non-diabetic patients.

No interim efficacy analyse will be performed so that no adjustment is required to the final $\mathrm{p}$ value to allow for the multiple testing. The DSMB will only analyse safety data and can make recommendation for adjustment of the number of patients to be included to ensure the statistical power of the mITT analysis. Important protocol modifications will be disseminated by the promotors to all the relevant parties.

\section{Method for missing data}

There should not be missing data for the primary outcome measure and the missing data rate should be low for the other outcomes as well. Missing data will be described by treatment arm. According to the rate of missing data (over 5\%), sensitivity analyses will be performed using multiple imputation methods as well as worst-case scenario (missing data considered as the most unfavourable case) and maximum bias scenario (missing data considered as the most favourable or unfavourable case in the placebo and experimental arms, respectively).

\section{Patient and public involvement statement}

The development of the research question and outcome measures was informed by patients' priorities, which is notably the prevention of postoperative complications. Patients were not involved in the design of the study. Patients were involved neither in the recruitment nor in the conduct of the study. Results of the study will be disseminated to study participants on personal request to the study coordinator. For this randomised clinical trial, the burden of the intervention was assessed by an institutional review board, notably composed of representative of patient associations.

\section{DISCUSSION}

The PACMAN trial is a nationwide randomised controlled study powered to investigate short course of moderate dose of dexamethasone in patients undergoing major non-cardiac surgery.

Several trials have investigated the benefits of administering corticosteroids in patients undergoing major surgery. However, there is yet no agreement on the beneficial effects of corticosteroids in alleviating surgical stress. This disagreement probably stems from the variability in the drugs used, their dosage and administration schedule, and the surgical procedures in different studies. Also, the fear of side effects induced by a possible immunosuppression (infections, postoperative wound complications, anastomotic leakage) explains the extreme variability of behaviour from one centre to another and among patients and physicians. These considerations prevented the performance of a large-scale randomised study despite evidences that corticosteroids could enhance outcome and even decrease the rate of infections after non-cardiac major surgery (see Introduction section). Before general recommendations for perioperative corticosteroids administration can be made, obviously conclusive safety studies must be available. So far, there seem to be no safety issues $^{22-24}$ related to a single preoperative dose of corticosteroids including specific studies on wound healing. ${ }^{25} 26$ The consequences of perioperative use of corticosteroids for glucose homoeostasis need further evaluation, but so far the transitory increased hyperglycaemic response 
has not been related to increased postoperative complications. ${ }^{27}$ To reach definitive conclusion on the effects of glucocorticoids on the risk of postoperative infections, we are eagerly awaiting the results from the PADDI (Perioperative ADministration of Dexamethasone and Infection Trial) randomised clinical trial which is investigating the effects of low dose of dexamethasone on surgical site infection, and from the PACMAN trial whose primary outcome is a composite outcome made of death and respiratory infections.

While perioperative glucocorticoids are widely administrated, the latest meta-analyses found that we are lacking power to detect differences in complications, ${ }^{11}$ and that no definitive conclusions can be made regarding clinically important benefice. ${ }^{7}$ While the PADDI study is a non-inferiority trial designed to test the safety of dexamethasone in an unselected population, we have designed the PACMAN trial to investigate the potential benefits of the treatment in a surgical population with high risk of postoperative respiratory complications according to American and European recommendations. ${ }^{12} 13$ Risk factors of pulmonary complications are either related to the surgery or to the medical history of the patients. The external validity of the PACMAN results to unselected patients should be cautious in case of increased risks of the side effects recorded as secondary outcomes.

We decided to not limit the PACMAN study to a specific type of surgery but to include all sort of surgery provided that the expected duration of the procedure exceeds $90 \mathrm{~min}$. This strategy maximises recruitment rates and improves the generalisation of results. We acknowledge that the clinical risk factors of postoperative respiratory complications are inconstantly used in clinical practice, which can limit the applicability to the study results. However, preoperative optimisation by nutritional support, respiratory physiotherapy or smoking cessation are recommended in patients presenting such risk factors. ${ }^{12}$ The selection of high-risk patients will result in a high incidence rate of the primary endpoint, which will result in a study of high clinical relevance and statistical power.

A wide range of doses of dexamethasone has been tested in surgical or critically ill patients: from low $\left(4-8 \mathrm{mg} /\right.$ day $\left.^{11}{ }^{28}\right)$, to high doses $\left(1-2 \mathrm{mg} / \mathrm{kg}^{6}{ }^{29}\right)$. The PACMAN trial is designed to investigate the effects of dexamethasone on the risk of major respiratory complications, notably pneumonia, and we thus decided to evaluate the effects of moderate doses as proposed in the latest recommendations and meta-analysis for the treatment with steroids of in patients with pneumonia. ${ }^{30} 31$ The timing of administration is also critical to consider. We decided to initiate the study treatment during the postoperative inflammatory response, rather than before the surgery, because glucocorticoids induce the apoptosis of immune cells in absence of inflammation but are stimulate immunity when administrated during an inflammatory response. ${ }^{32} 33$
We selected a composite outcome as the primary criteria. The use of mortality as a primary endpoint is never used in perioperative studies because the risk of death is low and the statistical power would be low. Respiratory complications are particularly frequent and serious after major surgery and we have recently proposed that glucocorticoid prevent the development of inflammation-related immunosuppression, ${ }^{8}$ and decrease the risk of pneumonia after severe trauma. ${ }^{9} 10$ Finally, mortality is not competitive with the primary criteria since the outcome all cause of death is included in the composite primary outcome.

This study has several limits. First, the primary outcome selected (composite of death, sepsis and pulmonary complications at 14 days) is biased towards the detection of benefit, as steroids are most likely to reduce pulmonary complications, but may have detrimental effects on other important outcomes, for example, wound infection. This problem will be partially compensated for by the extensive secondary outcome measures being collected. Second, for the primary statistical analysis of the primary outcome, we will use an mITT analysis including patients fulfilling all the inclusion criteria and who have received at least one injection of the experimental treatment. This strategy has recently been used in randomised clinical trials evaluating periprocedure treatments, ${ }^{17}{ }^{34}$ because the time between the randomisation, which is realised before the procedure, and the administration of the study treatment, which is realised during or after the procedure, expose the study to a high risk of included patients not receiving the allocated treatment (eg, cancelled or delayed surgery or perioperative complications). In this setting, we strongly believe that the mITT is more accurate to the medical field than the ITT. However, the exclusion of patients in the mITT analysis can theoretically decrease the statistical power of the study. Thus, the DSMB will have access to the number of patients excluded from the mITT analysis, and if necessary the DMSB will have the responsibility to propose an increase of the number of patients to be included to guarantee the study power. Finally, the ITT analysis will be reported in the final version of the manuscript.

\section{Trial status}

The trial has already achieved many milestones. Insurance for non-negligent harm has been provided by University Hospital of Nantes (France). The French Agency for the safety of medicines and medical devices authorised the study in September 2017 (\#170245RS-21). The current emphasis is on opening the recruitment infrastructures, which is ongoing and in developing the monitoring infrastructure. The study protocol was submitted to BMJ open before the first inclusion in the study. On 31 December 2018, 1050 patients have been included and randomised in the trial. The expected ending point of the study is February 2019.

The principal investigator (KA), the scientific expert $(\mathrm{EF})$, the statistician (FF) and the study coordinator 
(AR) will write the first draft of the manuscript. All the coauthors (investigators who had realised not less than 30 inclusions) will append and approve the final manuscript before the submission. No professional writer will be used.

In conclusion, the PACMAN trial is an investigator-initiated randomised controlled trial powered to test the hypothesis that the short course of moderate doses of dexamethasone in patients undergoing major non-cardiac surgery decreases the risk of postoperative complications. The results of the PACMAN trial will be relevant to the wide number of clinicians interested in perioperative medicine.

\section{Author affiliations}

${ }^{1}$ Anaesthesia and Intensive Care Unit, CHU Nantes, Nantes, France

${ }^{2}$ Anaesthesia and Intensive Care Unit, Centre Hospitalier Universitaire de ClermontFerrand, Clermont-Ferrand, France

${ }^{3}$ Plateforme de Biométrie, Direction de la Recherche, Département Promotion, CHU Nantes, Nantes, France

${ }^{4}$ INSERM, SPHERE U1246, Universite de Nantes, Nantes, France

${ }^{5} \mathrm{SAR}$, CHU Nantes, Nantes, France

Acknowledgements We thank patients and relatives, patient advisers, physicians, nursing staff and clinical research associates of the participating centres for their involvement in this important study.

Collaborators Collaborators in the alphabetical order of centres of the PACMAN group: Department of anesthesiology and critical care medicine Angers hospital (France): Sigismond Lasocki (MD,PhD), silasocki@chu-angers.fr. Department of anesthesiology and critical care medicine Brest Hospital (France): Olivier Huet (MD, PhD), olivier.huet@chu-brest.fr. Department of anesthesiology and critical care medicine Clermont Ferrrand hospital (France): Jean-Etienne Bazin (MD, PhD), jebazin@chu-clermontferrand.fr Department of anesthesiology and critical care medicine Beaujon Hospital (France), Catherine Paugam-Burtz (MD, PhD), catherine. paugam@bjn.aphp.fr. Department of anesthesiology and critical care medicine La Roche sur Yon Hospital (France): Gilbert Lorre (MD), anesthesie.rea@chd-vendee.fr. Department of anesthesiology and critical care medicine Le Mans Hospital (France) : Charlène LE Moal (MD), charlene.lemoal@orange.fr. Department of anesthesiology and critical care medicine Claude Huriez hospital, Lille (France) : Gilles Lebuffe (MD, PhD), gilles.lebuffe@chru-lille.f.r Department of anesthesiology and critical care medicine CHBS Scoff Hospital, (Lorient, France): Dr Guillaume Belliard (MD), g.belliard@ch-bretagne-sud.fr. Department of anesthesiology and critical care medicine Edouard Herriot hospital - University hospital of Lyon (France): Thomas Rimmele (MD), thomas.rimmele@chu-lyon.fr. Department of anesthesiology and critical care medicine Lyon Hospital, University Hospital of Lyon (France) : Vincent Piriou (MD, PhD), Vincent.piriou@chu-lyon.fr. Department of anesthesiology and critical care medicine Timone hospital - University hospital of Marseille (France), Nicolas Bruder (MD, PhD), nicolas.bruder@ap-hm.fr. Department of anesthesiology and critical care medicine Hôpital Nord - Assistance Publique-Hôpitaux de Marseille (France): Marc Leone (MD, PhD), marc.leone@ap-hm.fr. Department of anesthesiology and critical care medicine Institut Paoli Calmettes - Marseille (France) : Dr Djamel Mokart (MD), MOKARTD@ipc.unicancer.fr. Department of anesthesiology and critical care medicine B (DAR B) Saint-Eloi hospital - University hospital of Montpellier (France): Samir Jaber (MD, PhD), s-jaber@chu-montpellier. fr. Department of anesthesiology and critical care medicine Confluent Hospital Nantes (France): Nolwen Chatel-Josse (MD), Drchateljosee@ncn.fr. Department of anesthesiology and critical care medicine Laennec hospital - University hospital of Nantes (France) : Bertrand Rozec (MD, PhD), bertrand.rozec@chu-nantes.fr. Department of anesthesiology and critical care medicine Centre René Gauducheau Hospital Nantes (France) : Frederic Simonneau (MD), Frederic.simmoneau@ico. unicancer.fr. Department of anesthesiology and critical care medicine Clinic Jules Verne, Nantes (France): Florent Capron (MD), florent.capron@mla.fr. Department. of anesthesiology and critical care medicine Caremeau hospital - University hospital of Nîmes (France): Philippe Cuvillon (MD, PhD), philippe.cuvillon@chu-nimes.fr. Department of anesthesiology and critical care medicine Saint-Antoine hospital - Assistance Publique-Hôpitaux de Paris (AP-HP) (France) : Marc Beaussier (MD, $\mathrm{PhD}$ ), marc.beaussier@sat.aphp.fr Department of anesthesiology and critical care medicine Saint-Louis hospital - Assistance Publique-Hôpitaux de Paris (France) :
Benoit Plaud (MD, PhD), benoit.plaud@aphp.fr. Department of anesthesiology and critical care medicine Georges Pompidou Hospital - Assistance Publique-Hôpitaux de Paris (France) : Jean Mantz (MD, PhD), Jean.mantz@aphp.fr. Department of anesthesiology and critical care medicine Poitiers hospital, University Hospital of Poitiers (France) : Bertrand DEBAENNE (MD, PhD), b.debaene@chu-poitiers. fr. Department of anesthesiology and critical care medicine Robert Debre Hospital - University hospital of Reims (France) : Benny Charbit (MD, PhD), bcharbit@chureims.fr. Department of anesthesiology and critical care medicine Pontchaillou hospital - University hospital of Rennes (France) : Hélène Beloeil (MD, PhD), helene. beloeil@chu-rennes.fr. Department of anesthesiology and critical care medicine Charles Nicolle hospital - University hospital of Rouen (France) : Bertrand Dureuil (MD, PhD), Bertrand.Dureuil@chu-rouen.fr. Department of anesthesiology and critical care medicine Saint-Etienne University Hospital (France) : Serge Molliex (MD, PhD), serge.molliex@chu-st-etienne.fr. Department of anesthesiology and critical care medicine Nouvel Hôpital Civil - University teaching hospital of Strasbourg (France) : Dr Julien Pottecher (MD, PhD), julien.pottecher@chru-strasbourg. fr. Department of anesthesiology and critical care medicine Purpan hospital University hospital of Toulouse (France) : Thomas GEERAERTS (MD, PhD) geeraerts. t@chu-toulouse.fr

Contributors KA, EF, FF and AR conceived the study, coordinated its design and drafted the manuscript. AR and KA wrote the manuscript. EF and FF read and were involved in critical appraisal and revision of the manuscript. FF provided statistical expertise. All authors approved the final manuscript prior to submission.

Funding This study is an investigator-initiated trial, funded by a grant from the French Ministry of Health, grant number PHRCN 2016_0442.

Disclaimer The sponsor and the funder have no role in the design or conduct of the study, the data analysis, the writing of the manuscript or in the decision to submit the manuscript.

Competing interests None declared.

Patient consent for publication Not required.

Ethics approval Research ethics committee approval was obtained in September 2019 (Comité de Protection des Personnes Sud Méditerranée V).

Provenance and peer review Not commissioned; externally peer reviewed.

Open access This is an open access article distributed in accordance with the Creative Commons Attribution Non Commercial (CC BY-NC 4.0) license, which permits others to distribute, remix, adapt, build upon this work non-commercially, and license their derivative works on different terms, provided the original work is properly cited, appropriate credit is given, any changes made indicated, and the use is non-commercial. See: http://creativecommons.org/licenses/by-nc/4.0/.

\section{REFERENCES}

1. Weiser TG, Haynes AB, Molina G, et al. Estimate of the global volume of surgery in 2012: an assessment supporting improved health outcomes. Lancet 2015;385(Suppl 2):S11.

2. Pearse RM, Moreno RP, Bauer $\mathrm{P}$, et al. Mortality after surgery in Europe: a 7 day cohort study. Lancet 2012;380:1059-65.

3. Jhanji S, Thomas B, Ely A, et al. Mortality and utilisation of critica care resources amongst high-risk surgical patients in a large NHS trust. Anaesthesia 2008;63:695-700.

4. Jencks SF, Williams MV, Coleman EA. Rehospitalizations among patients in the Medicare fee-for-service program. N Engl J Med 2009;360:1418-28.

5. Asehnoune K, Hotchkiss RS, Monneret G. Understanding why clinicians should care about danger-associated molecular patterns. Intensive Care Med 2016;42:611-4.

6. Dieleman JM, Nierich AP, Rosseel PM, et al. Intraoperative high-dose dexamethasone for cardiac surgery: a randomized controlled trial. JAMA 2012;308:1761-7.

7. Whitlock RP, Devereaux PJ, Teoh KH, et al. Methylprednisolone in patients undergoing cardiopulmonary bypass (SIRS): a randomised, double-blind, placebo-controlled trial. Lancet 2015;386:1243-53.

8. Roquilly $A$, Broquet $A$, Jacqueline $C$, et al. Hydrocortisone prevents immunosuppression by interleukin-10+ natural killer cells after trauma-hemorrhage. Crit Care Med 2014;42:e752-61.

9. Roquilly A, Mahe PJ, Seguin P, et al. Hydrocortisone therapy for patients with multiple trauma: the randomized controlled HYPOLYTE study. JAMA 2011;305:1201-9.

10. Asehnoune K, Seguin P, Allary J, et al. Hydrocortisone and fludrocortisone for prevention of hospital-acquired pneumonia in patients with severe traumatic brain injury (Corti-TC): a double-blind, 
multicentre phase 3, randomised placebo-controlled trial. Lancet Respir Med 2014;2:706-16.

11. Toner AJ, Ganeshanathan V, Chan MT, et al. Safety of Perioperative Glucocorticoids in Elective Noncardiac Surgery: A Systematic Review and Meta-analysis. Anesthesiology 2017;126:234-48.

12. Smetana GW, Lawrence VA, Cornell JE, et al. Preoperative pulmonary risk stratification for noncardiothoracic surgery: systematic review for the American College of Physicians. Ann Intern Med 2006;144:581-95.

13. Kozek-Langenecker SA, Afshari A, Albaladejo P, et al. Management of severe perioperative bleeding: guidelines from the European Society of Anaesthesiology. Eur J Anaesthesiol 2013;30:270-382.

14. Weber WP, Mujagic E, Zwahlen M, et al. Timing of surgical antimicrobial prophylaxis: a phase 3 randomised controlled trial. Lancet Infect Dis 2017;17:605-14.

15. Futier E, Constantin JM, Paugam-Burtz C, et al. A trial of intraoperative low-tidal-volume ventilation in abdominal surgery. $N$ Engl J Med 2013;369:428-37.

16. Pearse RM, Harrison DA, MacDonald N, et al. Effect of a Perioperative, Cardiac Output-Guided Hemodynamic Therapy Algorithm on Outcomes Following Major Gastrointestinal Surgery. JAMA 2014;311:2181-10.

17. Futier E, Lefrant JY, Guinot PG, et al. Effect of Individualized vs Standard Blood Pressure Management Strategies on Postoperative Organ Dysfunction Among High-Risk Patients Undergoing Major Surgery: A Randomized Clinical Trial. JAMA 2017;318:1346-57.

18. Chanques $\mathrm{G}$, Conseil $\mathrm{M}$, Roger $\mathrm{C}$, et al. Immediate interruption of sedation compared with usual sedation care in critically ill postoperative patients (SOS-Ventilation): a randomised, parallelgroup clinical trial. Lancet Respir Med 2017;5:795-805.

19. Torres A, Niederman MS, Chastre J, et al. International ERS/ESICM/ ESCMID/ALAT guidelines for the management of hospital-acquired pneumonia and ventilator-associated pneumonia: Guidelines for the management of hospital-acquired pneumonia (HAP)/ventilatorassociated pneumonia (VAP) of the European Respiratory Society (ERS), European Society of Intensive Care Medicine (ESICM), European Society of Clinical Microbiology and Infectious Diseases (ESCMID) and Asociación Latinoamericana del Tórax (ALAT). Eur Respir J 2017; 50:1700582.

20. Leone M, Bouadma L, Bouhemad B, et al. Hospital-acquired pneumonia in ICU. Anaesth Crit Care Pain Med 2018;37:83-98.

21. Thompson JS, Baxter BT, Allison JG, et al. Temporal patterns of postoperative complications. Arch Surg 2003;138:596-602. discussion602-3.
22. Lunn TH, Kehlet H. Perioperative glucocorticoids in hip and knee surgery - benefit vs. harm? A review of randomized clinical trials. Acta Anaesthesiol Scand 2013;57:823-34.

23. Orci LA, Toso C, Mentha G, et al. Systematic review and metaanalysis of the effect of perioperative steroids on ischaemiareperfusion injury and surgical stress response in patients undergoing liver resection. Br J Surg 2013;100:600-9.

24. Holte K, Kehlet H. Perioperative single-dose glucocorticoid administration: pathophysiologic effects and clinical implications. $J$ Am Coll Surg 2002;195:694-712.

25. Wang AS, Armstrong EJ, Armstrong AW. Corticosteroids and wound healing: clinical considerations in the perioperative period. Am J Surg 2013;206:410-7.

26. Schulze S, Andersen J, Overgaard $\mathrm{H}$, et al. Effect of prednisolone on the systemic response and wound healing after colonic surgery. Arch Surg 1997;132:129-35.

27. Abdelmalak BB, Bonilla AM, Yang D, et al. The hyperglycemic response to major noncardiac surgery and the added effect of steroid administration in patients with and without diabetes. Anesth Analg 2013;116:1116-22.

28. Srinivasa S, Kahokehr AA, Yu T-C, T-c Y, et al. Preoperative Glucocorticoid Use in Major Abdominal Surgery. Ann Surg 2011;254:183-91.

29. Steinberg KP, Hudson LD, Goodman RB, et al. Efficacy and safety of corticosteroids for persistent acute respiratory distress syndrome. $N$ Engl J Med 2006;354:1671-84.

30. Stern A, Skalsky K, Avni T, et al. Corticosteroids for pneumonia. Cochrane Database Syst Rev 2017;385:1511-4.

31. Annane D, Pastores SM, Arlt W, et al. Critical illness-related corticosteroid insufficiency (CIRCI): a narrative review from a Multispecialty Task Force of the Society of Critical Care Medicine (SCCM) and the European Society of Intensive Care Medicine (ESICM). Intensive Care Med 2017;43:1781-92.

32. Lepelletier $\mathrm{Y}$, Zollinger $\mathrm{R}$, Ghirelli $\mathrm{C}$, et al. Toll-like receptor control of glucocorticoid-induced apoptosis in human plasmacytoid predendritic cells (pDCs). Blood 2010;116:3389-97.

33. Jamieson AM, Yu S, Annicelli $\mathrm{CH}$, et al. Influenza virus-induced glucocorticoids compromise innate host defense against a secondary bacterial infection. Cell Host Microbe 2010;7:103-14.

34. Weisbord SD, Gallagher M, Jneid H, et al. Outcomes after Angiography with Sodium Bicarbonate and Acetylcysteine. N Engl J Med 2018;378:NEJMoa1710933-12. 